\title{
New lower bound for multicolor Ramsey numbers for even cycles
}

\author{
Tomasz Dzido* \\ Institute of Mathematics, University of Gdańsk, \\ Wita Stwosza 57, 80-952 Gdańsk, Poland. \\ tdz@math.univ.gda.pl \\ Andrzej Nowik ${ }^{\dagger}$ \\ Institute of Mathematics, University of Gdańsk, \\ Wita Stwosza 57, 80-952 Gdańsk, Poland. \\ nowik@manta. univ.gda.pl \\ Piotr Szuca $a^{\ddagger}$ \\ Institute of Mathematics, University of Gdańsk, \\ Wita Stwosza 57, 80-952 Gdańsk, Poland. \\ pszuca@radix.com.pl
}

Submitted: Oct 5, 2004; Accepted: Jun 3, 2005; Published: Aug 30, 2005

Mathematics Subject Classifications: Primary 05C55; Secondary 05C15, 05C38

\begin{abstract}
For given finite family of graphs $G_{1}, G_{2}, \ldots, G_{k}, k \geq 2$, the multicolor Ramsey number $R\left(G_{1}, G_{2}, \ldots, G_{k}\right)$ is the smallest integer $n$ such that if we arbitrarily color the edges of the complete graph on $n$ vertices with $k$ colors then there is always a monochromatic copy of $G_{i}$ colored with $i$, for some $1 \leq i \leq k$. We give a lower bound for $k$-color Ramsey number $R\left(C_{m}, C_{m}, \ldots, C_{m}\right)$, where $m \geq 4$ is even and $C_{m}$ is the cycle on $m$ vertices.
\end{abstract}

\section{Introduction}

In this paper all graphs considered are undirected, finite and contain neither loops nor multiple edges. By $K_{m}$ we denote the complete graph on $m$ vertices, and by $C_{m}$ we denote the cycle of length $m$. For given graphs $G_{1}, G_{2}, \ldots, G_{k}, k \geq 2$, the multicolor

\footnotetext{
${ }^{*}$ The first author was partially supported by KBN grant 4 T11C 04725 .

${ }^{\dagger}$ The second author was partially supported by grant BW/5100-5-0145-4.

${ }^{\ddagger}$ The third author was partially supported by grant BW/5100-5-0095-5.
} 
Ramsey number $R\left(G_{1}, G_{2}, \ldots, G_{k}\right)$ is the smallest integer $n$ such that if we arbitrarily color the edges of the complete graph of order $n$ with $k$ colors, then it always contains a monochromatic copy of $G_{i}$ colored with $i$, for some $1 \leq i \leq k$. We denote such a number by $R_{k}(G)$ if $G=G_{1}=G_{2}=\cdots=G_{k}$. Here in, we consider only 3-color Ramsey number $R_{3}(G)$ (i.e. we color the edges of the complete graph $K_{n}$ with color red, blue and green.) A 3-coloring of $K_{n}$ is called a $(G ; n)_{3}$-coloring if it contains neither a red $G$ nor a blue $G$ nor a green $G,(G ; n)_{k}$-coloring is defined analogously. We refer the reader to [6] for a survey.

\section{The Ramsey numbers for even cycles}

Up to now, there have been known only two exact values for 3-color Ramsey numbers for even cycles. More precisely, in [2] it was proved that $R_{3}\left(C_{4}\right)=11$, and $R_{3}\left(C_{6}\right)=12$ was showed in [7] with a help of the computer support. When talking about lower bounds, let us recall that Graham et al. [5] proved that for any $k$ and $m, R_{k}\left(C_{2 m}\right) \geq(k-1)(m-1)+$ 1. This bound was improved to $(k+1) m-k+1$ in [3]. Finally, recall that Figaj and Euczak proved the following theorem.

Theorem 1 ([4]). For any constants $\alpha_{1}, \alpha_{2}, \alpha_{3}>0$,

$$
R\left(C_{2\left\lfloor\alpha_{1} n\right\rfloor}, C_{2\left\lfloor\alpha_{2} n\right\rfloor}, C_{2\left\lfloor\alpha_{3} n\right\rfloor}\right)=\left(\alpha_{1}+\alpha_{2}+\alpha_{3}+\max \left\{\alpha_{1}, \alpha_{2}, \alpha_{3}\right\}+o(1)\right) n
$$

while $n \rightarrow \infty$.

Consequently, notice that if $\alpha_{1}=\alpha_{2}=\alpha_{3}=1$ and $n=m$ we obtain that

$$
R_{3}\left(C_{2 m}\right)=(4+o(1)) m .
$$

In this paper, our main result is the following theorem.

Theorem 2. For all integers $m \geq 2$ and an odd integer $k \geq 1$,

$$
R_{k}\left(C_{2 m}\right) \geq(k+1) m \text {. }
$$

Proof. We shall give a $k$-coloring of all edges of a complete graph $G^{\prime \prime}=K_{n}$ on $n=$ $(k+1) m-1$ vertices which is a $\left(C_{2 m} ; n\right)_{k}$-coloring. The situation for $k=1$ is obvious, so we may assume that $k \geq 3$.

Let $k \geq 3$ be an odd integer. Using a fact that $\chi^{\prime}\left(K_{k+1}\right)=k$ when $k$ is odd (see e.g. [8]), color properly edges of the complete graph $K_{k+1}$ with $k$ colors. "Blow-up" the coloring $m-1$ times, i.e. replace each vertex of $K_{k+1}$ by the set $G_{i}(1 \leq i \leq k+1)$ of $m-1$ vertices and each colored edge by a complete monochromatic bipartite graph $K_{m-1, m-1}$ of an appropriate color (See Fig. 1 for illustration.) Formally, consider the complete graph $G$ on $k+1$ vertices. Let $c: V \rightarrow\{1, \ldots, k+1\}$ be a proper edge-coloring of graph $G$. For a vertex $i \in V$, where $i \in\{1, \ldots, k+1\}$, let $G_{i}$ denote a complete graph on $m-1$ vertices. 


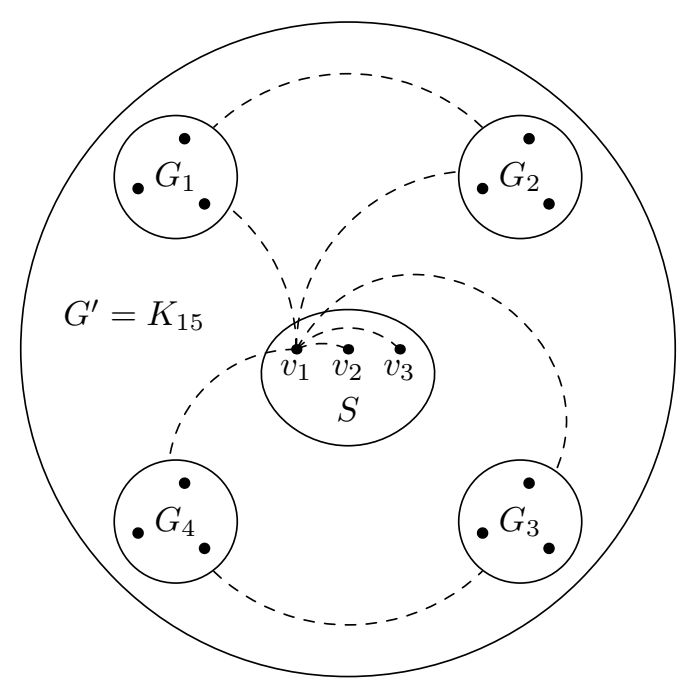

Figure 1: An illustration of coloring from the proof of Theorem 2 for the case $m=4$ and $k=3$. To make the picture readable there are shown only edges colored with color 1: ones which join $v_{1}$ with each $G_{i}$, and edges joining $G_{1}$ with $G_{2}$, and $G_{3}$ with $G_{4}$. Edges which join $v_{2}$ with each $G_{i}, G_{2}$ with $G_{3}$, and $G_{1}$ with $G_{4}$ are colored with color 2 . Edges which join $v_{3}$ with each $G_{i}, G_{1}$ with $G_{3}$ and $G_{2}$ with $G_{4}$ are colored with color 3. Subgraphs $G_{1}, G_{2}, G_{3}$ and $G_{4}$ replace "blown-up" vertices of the complete graph $K_{4}$.

Let $G^{\prime}\left(V^{\prime}, E^{\prime}\right)$ be a complete graph with the set of vertices

$$
V^{\prime}=\bigcup_{i=1}^{k+1} V\left(G_{i}\right)
$$

The coloring $c^{\prime}$ of the graph $G^{\prime}$ is as follows:

$$
c^{\prime}(\{p, q\})= \begin{cases}c(x, y) & \text { if } p \in V\left(G_{x}\right), \text { and } q \in V\left(G_{y}\right), \text { and } x \neq y \\ 1 & \text { otherwise. }\end{cases}
$$

Obviously, such a graph $G^{\prime}$ contains no monochromatic path of more than $2 m-2$ vertices.

Next, extend graph $G^{\prime}$ to the graph $G^{\prime \prime}$ by adding $k$ new vertices $S=\left\{v_{1}, \ldots, v_{k}\right\}$. Now, color all edges between $v_{i}$ and $V^{\prime}$ with the color $i$, and for any pair $\{i, j\}$ such that $j>i>0$, color edge $\left\{v_{i}, v_{j}\right\}$ with the color $i$ (thus there are no monochromatic cycles in the subgraph induced by $S$.)

More formally, let $G^{\prime \prime}\left(V^{\prime \prime}, E^{\prime \prime}\right)$ be a complete graph with the set of vertices $V^{\prime \prime}=V^{\prime} \cup S$. The coloring $c^{\prime \prime}$ of $G^{\prime \prime}$ is as follows:

$$
c^{\prime \prime}(\{p, q\})= \begin{cases}i & \text { if } p=v_{i} \in S, \text { and } q \in V\left(G_{j}\right), \text { and } 1 \leq j \leq k+1 \\ i & \text { if } p=v_{i} \in S, \text { and } q=v_{j} \in S, \text { and } 1 \leq i<j \leq k \\ c^{\prime}(\{p, q\}) & \text { otherwise. }\end{cases}
$$

It remains to show that in $G^{\prime \prime}$ there are no monochromatic cycles of length at least $2 m$. Suppose, contrary to our claim, that $G^{\prime \prime}$ contains a cycle $C$ of color $d$ longer than 


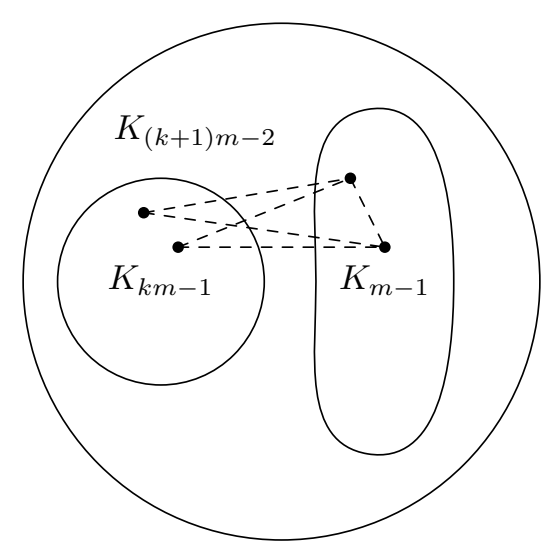

Figure 2: An illustration of coloring from the proof of Corollary 1. Edges of $K_{k m-1}$ are colored with $k-1$ colors without monochromatic cycle of length $2 m$. All edges from the graph $K_{m-1}$ and a bipartite graph $K_{k m-1, m-1}$ are colored with color $k$ (dashed line denote edges assigned with color $k$.)

$2 m-1$. Since in $G^{\prime}$ there is no monochromatic path of length greater than $2 m-2$, we have

$$
C \cap S \neq \emptyset \text {. }
$$

Next, the only vertex from $S$ which is adjacent by an edge of color $d$ with $G$ is $v_{d}$, hence

$$
C \cap S=\left\{v_{d}\right\}
$$

Since $v_{d}$ can be contained only once in the cycle $C$, this implies that the cardinality of the set

$$
\left\{i: C \cap G_{i} \neq \emptyset\right\}
$$

is at most 2. Thus the length of $C$ is less than $2 m$, a contradiction.

Corollary 1. For all integers $m \geq 2$ and an even integer $k \geq 2$,

$$
R_{k}\left(C_{2 m}\right) \geq(k+1) m-1
$$

Proof. Let $n=(k+1) m-2$. By Theorem 2, there exists $\left(C_{2 m} ; k m-1\right)_{k-1}$-coloring of a complete subgraph $K_{k m-1}$ of $K_{n}$. A $\left(C_{2 m} ; n\right)_{k}$-coloring of $K_{n}$ is obtained by assigning the last color $k$ to all remaining edges (See Fig. 2.) Indeed, on the contrary suppose that there exists a monochromatic cycle of length $2 \mathrm{~m}$. This cycle has the last $k$-th color. The number of vertices from $K_{k m-1}$ which belong to cycle is at least $m+1$ and is greater than the number of such vertices from $K_{m-1}$. The maximal possible number of edges between $K_{k m-1}$ and $K_{m-1}$ is $2 m-2$. Thus there exists an edge contained in the graph $K_{k m-1}$, what is impossible.

The following corollary is straightforward: 
Corollary 2. For all integers $m \geq 2$,

$$
R_{3}\left(C_{2 m}\right) \geq 4 m
$$

In particular, notice that we obtain $R_{3}\left(C_{8}\right) \geq 16$. Moreover, by using upper bound for Ramsey number for even cycles ([5, Section 5.7, Theorem 10]), we have $R_{3}\left(C_{8}\right) \leq 2412$, and by using known upper bound for Ramsey number for symmetric bipartite graph $K_{4,4}$ ([1]), we have $R_{3}\left(C_{8}\right) \leq 648$.

\section{Acknowledgment}

We would like to thank the anonymous referees for many helpful suggestions, especially for the simplified proof of Theorem 2. Also we would like to express our gratitude to Professor Paweł Żyliński for his critical reading of the manuscript and for pointing out a number of corrections.

\section{References}

[1] Chvátal, V., Harary, F.: Generalized Ramsey theory for graphs. I. Diagonal numbers, Periodica Mathematica Hungarica, 3 (1973) 115-124.

[2] Clapham C.: The Ramsey Number $R\left(C_{4}, C_{4}, C_{4}\right)$, Periodica Mathematica Hungarica, 18 (1987) 317-318.

[3] Dzido T.: Multicolor Ramsey numbers for paths and cycles, Discussiones Mathematicae Graph Theory, 25 (2005) 57-65.

[4] Figaj A., Łuczak T.: The Ramsey number for a triple of long even cycles (to appear).

[5] Graham R.L., Rothschild B.L., Spencer J.H.: Ramsey Theory (2nd ed) (John Wiley and Sons 1990).

[6] Radziszowski S.P.: Small Ramsey Numbers, Electronic Journal of Combinatorics, Dynamic Survey 1, revision \#10, July 2004, http://www.combinatorics.org.

[7] Rowlison P., Yang Y.: On Graphs without 6-Cycles and Related Ramsey Numbers, Utilitas Mathematica, 44 (1993) 192-196.

[8] Wilson R.J.: Introduction to graph theory (3rd ed) (Longman 1985). 